\title{
Can Incarceration of Uterus between 14 - 17 Wks. Be Managed by General Anaesthesia Only? A Second Reported Case
}

\author{
Divyesh V. Shukla*, Shilpi D. Shukla, Amit Shah, Yesha Choksi \\ Isha Hospital, Sarabhai Campus, Vadodara, India \\ Email: *divyeshshukla@yahoo.com, ishahospital.com
}

How to cite this paper: Shukla, D.V., Shukla, S.D., Shah, A. and Choksi, Y. (2018) Can Incarceration of Uterus between $14-17$ Wks. Be Managed by General Anaesthesia Only? A Second Reported Case. Open Journal of Obstetrics and Gynecology, 8, 720-727.

https://doi.org/10.4236/ojog.2018.88076

Received: June 5, 2018

Accepted: July 8, 2018

Published: July 11, 2018

Copyright $(0) 2018$ by authors and Scientific Research Publishing Inc. This work is licensed under the Creative Commons Attribution International License (CC BY 4.0).

http://creativecommons.org/licenses/by/4.0/

\begin{abstract}
This is a case of primigravida of 35 years of age having spontaneous conception \& 14.5 wks. pregnancy with retention of urine and pain in lower abdomen for 5 days. There was history of unsuccessful attempt of correction of retroversion one day prior. Transabdominal USG (ultrasonography) confirmed 14.5 wks. live pregnancy with incarceration of uterus. In this case, only GA (general anaesthesia) corrected retroversion \& incarceration.
\end{abstract}

\section{Keywords}

Incarceration, USG, GA, Retention of Urine, 14 - 20 Wks. Pregnancy

\section{Background}

Uterine incarceration is an unexpected complication in the $2^{\text {nd }}$ trimester of pregnancy irrespective of parity. Predisposing factors as pelvic adhesions, fundal fibroids, septate uterus, bicornuate uterus, deep sacral cavity with overhanging sacral promontory may go unnoticed when patient comes with pregnancy [1] [2] [3] [4]. Uterine incarceration can also occur in the absence of the predisposing factors [5]. We see about $11 \%$ patients with retroverted uterus in the first trimester of pregnancy without problem. Spontaneous correction of the uterus to anteversion occurs between 12 and 16 wks., but in 1.4\% this does not occur [1] [6].

In some of these cases, retroverted uterus becomes incarcerated in the pelvic cavity, and the cervix is displaced anteriorly. This causes elongation of the urethra and compression of the bladder neck against pubic symphysis and urinary retention. Unrecognized incarceration of uterus may cause premature rupture of membrane, the $2^{\text {nd }}$ trimester abortion \& preterm delivery. Serious maternal and 
fetal complications such as bladder rupture, urinary retention, hydronephrosis, and renal failure, result from compression of the bladder and urethra, as well as uterine rupture and uterine wall necrosis resulting from excess stretching of the uterine wall, rectal gangrene, cervicovaginal fistula, and thrombosis [7] [8] [9] [10].

\section{Case Presentation}

A 35 years primigravida with Ht: $168 \mathrm{~cm}$, Wt: $62 \mathrm{~kg}$, BMI: 22 with 14.5 wks. pregnancy presented with acute retention of urine for 5 days for which self-retaining catheter was inserted. There was associated lower abdominal pain which was not relieved. She had spontaneous conception \& there was no significant past history. There was history of failed attempt of manual correction of retroversion without anaesthesia 1 day back. On examination her vitals were, pulse: $84 / \mathrm{min}$, B.P.: $120 / 70 \mathrm{~mm}$ of $\mathrm{Hg}, \mathrm{SpO}_{2}$ : $99 \%$. Per abdominal examination uterus was not palpable. There was tenderness in lower abdomen. She refused per speculum \& prevaginal examination because of pain. Trans abdominal USG had findings of single live intrauterine pregnancy of 14.5 wks. maturity. No gross malformations seen. Cervix flat anteriorly placed (red arrow) with bladder in front of it (white arrow) with fundus of the uterus in sacral hollow (green arrow). Posterior wall of uterus is in contact with posterior cervix (yellow arrow). (Figures 1-3) [8]. Additionally, we reported thin uterine walls (thickness compared with wall after correction). No adnexal mass or pelvic mass or uterine torsion was seen.MRI was not done as diagnosis was clear. Her blood investigations were normal but urine microscopy examination reported 20 - 25 pus cells. Urine culture had E coli isolated with colony count $>100,000 \mathrm{CFU} / \mathrm{ml}$. Antibiotics were started as per sensitivity report. Antibiotic sensitivity report revealed sensitivity to aminoglycosides \& carbapenems and resistant to penicillin, cephalosporins, b-lactamase inhibitors and quinolones. Patients were treated by intravenous amikacin in dose of $500 \mathrm{mg}$ bd for 7 days.

Patient was scheduled after counselling for spontaneous correction or manual correction or laparoscopic surgery under GA depending upon the weather incarceration gets corrected before invasive procedure (laparoscopy) on the next day of admission. Patient was kept fasting overnight. In the operation theatre patient was given lithotomy position in awake state and painting and draping was done. GA was gradually instituted.

\section{Protocol for GA}

Standard monitoring of ECG, NBP, $\mathrm{SPO}_{2}$, TEMP, RR was applied and premedication of Injection glycopyrrolate $0.2 \mathrm{mg}$ and Injection Fentanyl $60 \mathrm{mcg}(1$ $\mathrm{mcg} / \mathrm{kg}$ ) was given IV. Nasal $\mathrm{O}_{2}$ was started.

Patient was pre-oxygenated with $100 \%$ oxygen for 3 minutes. Patient was induced with $120 \mathrm{mg}$ of propofol $(2 \mathrm{mg} / \mathrm{kg})$. Following loss of consciousness and relaxation of jaw, Igel (supraglottic airway) size 4 was inserted as per manufacturer's instructions. After insertion of Igel pressure-controlled ventilation started 


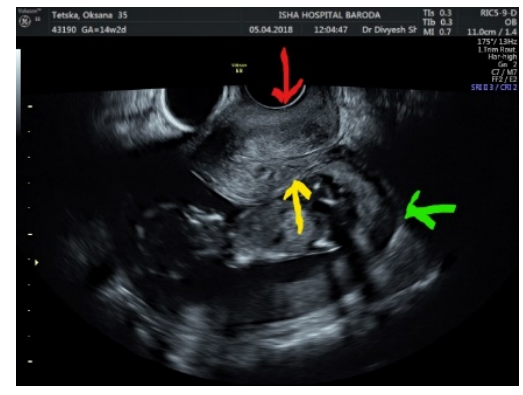

Figure 1. TAS, 15 wks. pregnancy position of cervix (red arrow), fundus of the uterus (green arrow) and posterior uterine wall (yellow arrow). Note stretched thin uterine wall.

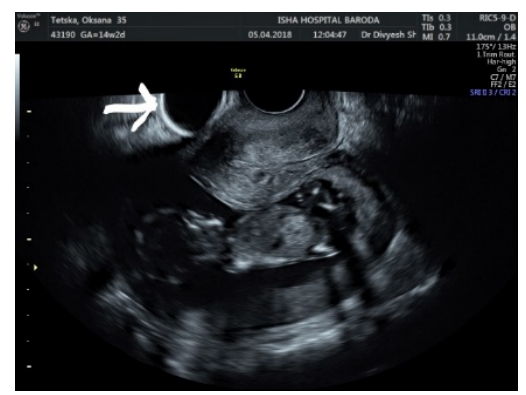

Figure 2. TAS, 15 wks. pregnancy, showing bladder in front of cervix, foley'scatheter's bulb seen (white arrow).

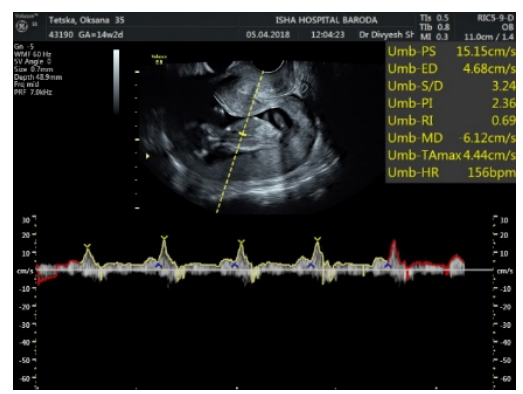

Figure 3. TAS, 15 wks. live pregnancy.

with inspiratory pressure of 12 and Peep of $5 \mathrm{~cm}$ of $\mathrm{H}_{2} \mathrm{O}$ since patient had already develop apnoea. Oxygen flow was kept at $6 \mathrm{~L}$, and Sevoflurane dial was kept at $8 \%$. Within 4 - 5 minutes end tidal value reached 4\% (2 MAC for sevoflurane). At this point Incarceration was corrected immediately. It was confirmed by USG and sevoflurane was switched off immediately and patient was allowed to wake up. Patent woke up immediately within 10 - 15 minutes (Table $1)$.

Meanwhile per speculum examination and TAS USG was performed simultaneously.

Per speculum examination revealed anteriorly placed cervix gradually shifting to mid position \& TAS USG dynamically witnesses correction of incarceration with shift of cervix to in its mid position and returning back the thickness of uterine walls (Figures 4-9). Self-retaining catheter was removed. 


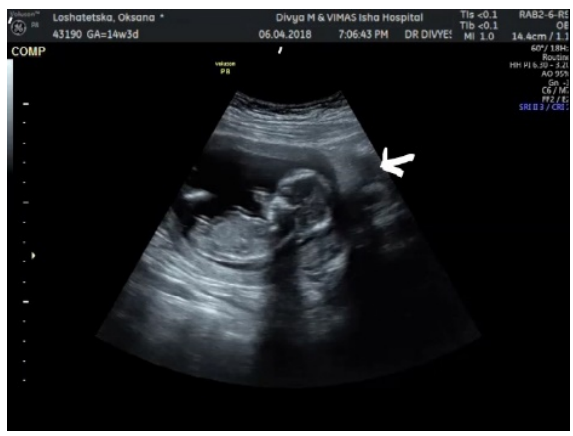

Figure 4. TAS showing change in position of cervix (white arrow) and fundus of the uterus under anaesthesia.

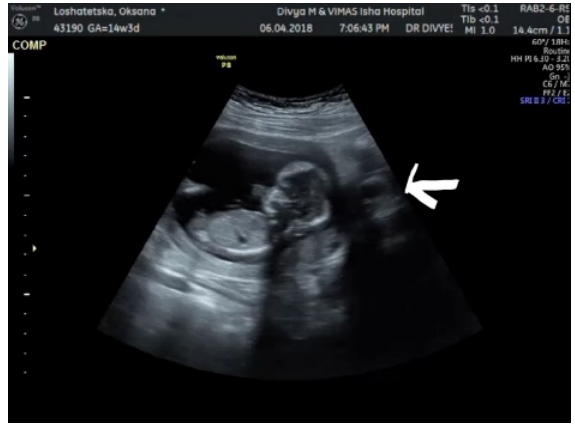

Figure 5. TAS, shows further change in cervical position (white arrow) under anaesthesia.

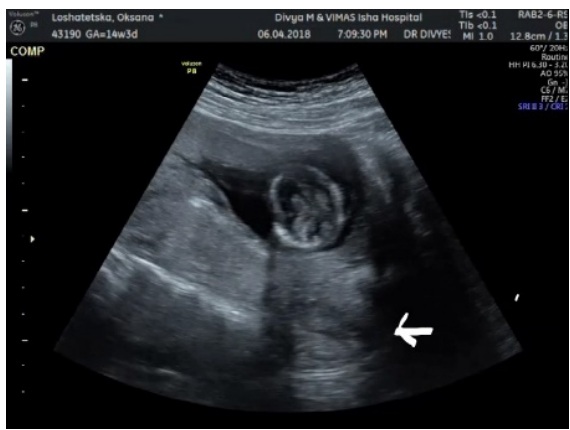

Figure 6. TAS, shows normal position of cervix (white arrow) and fundus of the uterus and return of normal thickness of uterine wall.

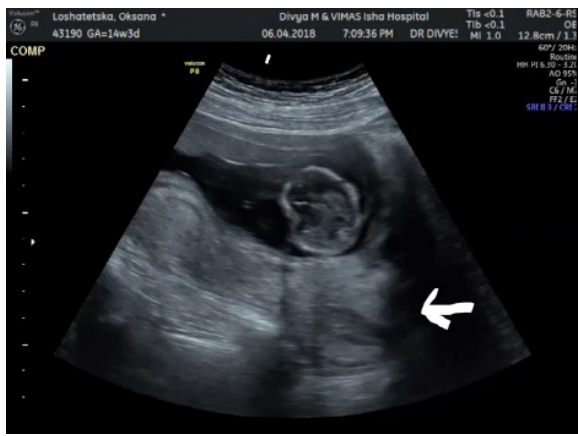

Figure 7. TAS, Complete return of normal cervical position (white arrow). 


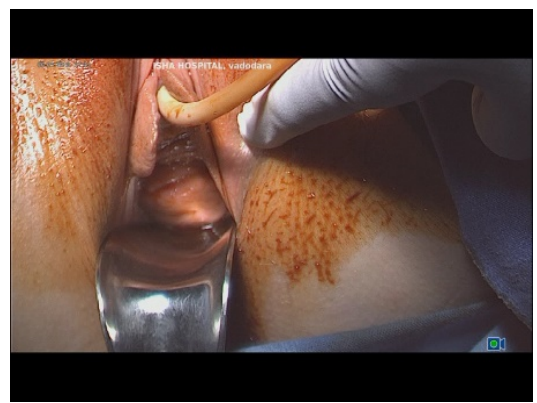

Figure 8. Cervix not seen before complete relaxation of uterus on per speculum examination.

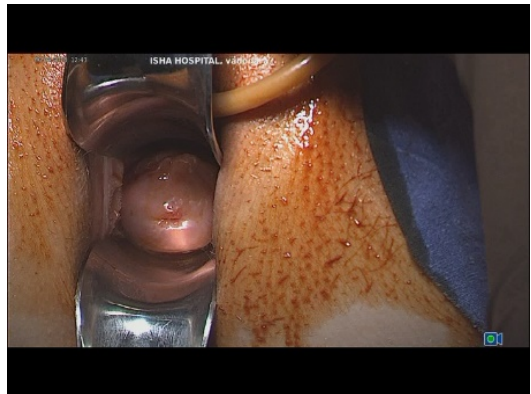

Figure 9. Cervix seen spontaneously on correction of retroversion after complete uterine relaxation on per speculum examination.

Table 1. Inhalation agent (sevoflurane) concentration used for uterine relaxation.

\begin{tabular}{cccc}
\hline Time & Oxygen flow & Dial sevoflurane & End tidal concentration \\
\hline $12.35 \mathrm{pm}$ & 10 L pre-oxygenation & & \\
$12.36 \mathrm{pm}$ & Insertion of Igel and start of ventilation & & \\
12.37 & $6 \mathrm{~L}$ & $8 \%$ & - \\
12.38 & $6 \mathrm{~L}$ & $8 \%$ & $1.5 \%$ \\
12.39 & $6 \mathrm{~L}$ & $8 \%$ & $2 \%(1 \mathrm{MAC}$ Value $)$ \\
12.40 & $6 \mathrm{~L}$ & $8 \%$ & $3 \%$ \\
12.41 & $6 \mathrm{~L}$ & $8 \%$ & $4 \%(2 \mathrm{MAC}$ Value $)$ \\
12.42 & $6 \mathrm{~L}$ Incarceration corrected-confirmed & $8 \%$ & $4 \%$ \\
12.43 & $6 \mathrm{~L}$ rechecked & 0 & $3 \%$ \\
12.44 & $6 \mathrm{~L}$ & 0 & $2 \%$ \\
12.45 & $6 \mathrm{~L}$ & 0 & $1 \%$ \\
12.46 & $6 \mathrm{~L}$ & 0 & $0.3 \%$ \\
\hline
\end{tabular}

Patient was discharged on same day after she could pass urine on catheter removal. Antibiotics were given for 7 days for UTI. Post-operative tocolysis with nifedipine as per standard protocol was given for $72 \mathrm{hrs}$. She was advised to practise muslim prayer position or repeated knee-elbow position (Simm's position) for multiple times in a day up to 20 wks. of pregnancy. 


\section{Follow-Up}

Patient had no complaints in follow up visits. UTI was cured. When we are reporting this case, she has 34 wks. ongoing uneventful pregnancy.

\section{Discussions}

Pregnant women diagnosed with a retroverted uterus in the $1^{\text {st }}$ trimester of pregnancy, should have repeat pelvic examination and USG in the $2^{\text {nd }}$ trimester [11]. Usually spontaneous correction occurs by 14 wks. of pregnancy. When patients have urinary problem in second trimester, uterine position should be checked. Once incarceration is diagnosed, immediate correction should be done in the $2^{\text {nd }}$ trimester. The incidence of incarcerated uterus has been quoted to be around 1 in 3000 to 10,000 cases [1]. In my clinical experience of 26 years with total no of institutional deliveries of about 68,000 in number, this was the $1^{\text {st }}$ case of incarceration treated.

As it is rare, management depends on the methods reported by various published reports adopted for that wk. for pregnancy.

Most authors report repositioning of the uterus up to $15 \mathrm{wks}$. beyond which if attempted, more failures \& spontaneous abortions are reported [12].

We report spontaneous correction of incarceration following general anaesthesia in $15^{\text {th }}$ wk. of pregnancy without manual assistance which was dynamically recorded on ultrasound. Protocol for anaesthesia is mentioned with sevoflurane concentration which completely relaxes the uterus and results in spontaneous correction of incarceration. Withdrawal of anaesthesia leads to return of polarity of uterus to normal. Simultaneous per speculum \& prevaginal examination witnessed shift of cervix from anterior to mid position \& fullness of fornix to disappear. This is the second such case reported in the literature. In this patient, incarceration could be due to persistent retroversion. Patient had deep sacral curve \& prominent sacral promontory which was felt on per vaginal examination under anaesthesia. This may have predisposed to incarceration. Pelvic findings were not documented by MRI in this patient [13]. The first such case of using general anaesthesia only to correct uterine incarceration was reported in 2015 Dec. [14]. Use of general anaesthesia \& USG guided manual reduction has been reported at 20 wks. of pregnancy [15]. GA causes relaxation of uterine \& skeletal muscles of pelvis which may lead to spontaneous correction of uterine axis.

We believe only giving general anaesthesia may be tried to correct incarceration diagnosed up to 20 wks. This should be done if manual attempt to correct incarceration without using anaesthesia fails.

In cases of incarceration diagnosed at 17 - 18 wks. of pregnancy, other method of treatment by a colonoscopy-assisted manipulation of uterus without anaesthesia with the woman lying in a left lateral decubitus position, followed by a left lateral tilted supine position, is described. Simultaneously, with the insertion of the flexible sigmoidoscope, an extra anterior pressure was generated by insertion 
of two fingers in the vagina. Ensure bladder and bowel are empty [16] [17].

Rarely an incarcerated gravid uterus resolves spontaneously even in the $3^{\text {rd }}$ trimester of pregnancy. Severe abdominal pain might be caused by spontaneous reduction [18].

\section{Learning Points}

GA with inhalation anaesthetics only can be adequate in treating incarcerated uterus diagnosed from 14 - 17 wks. of pregnancy.

\section{Conflict of Interests}

There was no conflict of interest \& due consent of the patient was taken to report her clinical case.

\section{References}

[1] Schwartz, Z., Dgani, R., Katz, Z. and Lancet, M. (1986) Urinary Retention Caused by Impaction of Leiomyoma in Pregnancy. Acta Obstetricia et Gynecologica Scandinavica, 65, 525-526. https://doi.org/10.3109/00016348609157402

[2] Smith, J.J., Schwartz, E.D. and Romney, S.L. (1962) Anterior Sacculation of the Pregnant Uterus. Obstetrics \& Gynecology, 20, 536-538.

[3] Evans, A.J., Anthony, J. and Masson, G.M. (1986) Incarceration of the Retroverted Gravid Uterus at Term. Case Report. British Journal of Obstetrics and Gynaecology, 93, 883-885. https://doi.org/10.1111/j.1471-0528.1986.tb07999.x

[4] Vleugels, M.P.H. and Meuwissen, J.H.J.M. (1987) Confusing Presentation in a Retroflexed Septate Uterus at Term. European Journal of Obstetrics \& Gynecology and Reproductive Biology, 24, 237-241.

[5] Weekes, A.R.L., Atlay, R.D., Brown, V.A., Jordan, E.C. and Murray, S.M. (1976) The Retroverted Gravid Uterus and Its Effect on the Outcome of Pregnancy. British Medical Journal, 1, 622-624. https://doi.org/10.1136/bmj.1.6010.622

[6] Goldberg, K.A. and Kwart, A.M. (1981) Intermittent Urinary Retention in First Trimester of Pregnancy. Urology, 17, 270-271. https://doi.org/10.1016/0090-4295(81)90047-9

[7] Newell, S.D., Crofts, J.F. and Grant, S.R. (2014) The Incarcerated Gravid Uterus: Complications and Lessons Learned. Obstetrics \& Gynecology, 123, 423-427. https://doi.org/10.1097/AOG.0000000000000102

[8] Gardner, C.S., Jaffe, T.A., Hertzberg, B.S., Javan, R. and Ho, L.M. (2013) The Incarcerated Uterus: A Review of MRI and Ultrasound Imaging Appearances. American Journal of Roentgenology, 201, 223-229. https://doi.org/10.2214/AJR.12.9473

[9] Sweigart, A. and Matteucci, M. (2008) Fever, Sacral Pain and Pregnancy: An Incarcerated Uterus. The Western Journal of Emergency Medicine, 9, 232-234.

[10] Singh, M.N., Payappagoudar, J., Lo, J. and Prashar, S. (2007) Incarcerated Retroverted Uterus in the Third Trimester Complicated by Postpartum Pulmonary Embolism. Obstetrics \& Gynecology, 109, 498-501. https://doi.org/10.1097/01.AOG.0000218695.71256.cf

[11] Gibbons, J.M. and Paley, W.B. (1969) The Incarcerated Gravid Uterus. Obstetrics \& Gynecology, 33, 842-845.

[12] Van Winter, J.T., Ogburn Jr., P.L., Ney, J.A. and Hetzel, D.J. (1991) Uterine Incar- 
ceration during the Third Trimester: A Rare Complication of Pregnancy. Mayo Clinic Proceedings, 66, 608. https://doi.org/10.1016/S0025-6196(12)60520-5

[13] Van Beekhuizen, H.J., Bodewes, H.W., Tepe, E.M., et al. (2003) Role of Magnetic Resonance Imaging in the Diagnosis of Incarceration of the Gravid Uterus. Obstetrics \& Gynecology, 102, 1134. https://doi.org/10.1097/00006250-200311001-00004

[14] Ramli, R., et al. (2015) Spontaneous Repositioning of Incarcerated Gravid Uterus Following General Anaesthesia: A Case Report and Review of Literature. International Journal of Reproduction, Contraception, Obstetrics and Gynecology, 4, 2041-2204.

[15] Grossenburg, N.J., Delaney, A.A. and Berg, T.G. (2011) Treatment of a Late Second-Trimester Incarcerated Uterus Using Ultrasound-Guided Manual Reduction. Obstetrics \& Gynecology, 118, 436-439.

[16] Seubert, D.E., Puder, K.S., Goldmeier, P. and Gonik, B. (1999) Colonoscopicrelease of the Incarcerated Gravid Uterus. Obstetrics \& Gynecology, 94, 792-794.

[17] Dierickx, I. and Delens, F. (2014) Case Report: Incarceration of the Gravid Uterus: A Radiologic and Obstetric Challenge. Journal of Radiology Case Reports, 8, 28-36.

[18] Takami, M. and Hasegawa, Y. (2016) Spontaneous Reduction of an Incarcerated Gravid Uterus in the Third Trimester. Clinical Case Reports, 4, 605-610. https://doi.org/10.1002/ccr3.577 